\title{
80. ON THE RELATION BETWEEN COMETS AND METEOROIDS
}

\author{
H. ALFVÉN \\ Royal Institute of Technology, Stockholm, Sweden
}

In order to understand the origin and evolution of the solar system it is especially important to study the small bodies - asteroids, comets and meteoroids - because in the planetesimal state (preceding the present state) the matter was dispersed.

An essential part of the cosmogonic problem is the state of the primeval plasma and how small bodies condensed from it. This directs the attention to plasma physics, which has developed very rapidly because of thermonuclear research. Plasma physics deals with the interaction of a large number of bodies. Many of its theorems are applicable also to the motion of neutral (solid) particles in a Coulomb field, and we can therefore make use of them when studying the behaviour of small particles in interplanetary space.

One of the results is that the interaction of particles will lead to the formation of jet streams, i.e., the particles will tend to move in similar orbits. This is contrary to the general belief that encounters produce a state of random motion. Some support for the jet-stream theory is provided by the results by Arnold and Danielsson that such streams exist in the asteroid belt.

In principle, meteor streams could be produced by the jet-stream mechanism. We thus have an alternative to the usual theory, and this alternative ought to be studied further. We can then proceed a step further and ask whether comets may be produced from jet streams. We can again take an analogy from plasma physics. An electron beam acted on by a variable electric field will 'bunch' so that its density in some regions will increase by many orders of magnitude. Hence a condensation could be formed in a meteor stream. It is desirable to explore whether the formation of comets may be explained in this way. The matter is especially interesting in the case of the short-period comets, for there seems to be little chance of explaining these comets on the basis of the capture theory.

\section{Discussion}

F. L. Whipple: The idea of meteor stream condensation is interesting, but I believe it is quite impossible in the present solar system - although it may have been important in the formation of the solar system. Meteoric particles are dispersed by gas when ejected by comets; further, they are dispersed by the differences in the orbital elements, especially the period, and because of the differential perturbations by the planets. They are embedded in a high-temperature medium, and this causes frequent collisions and dissipation. Solar radiation pressure quickly removes the fine particles (those with dimensions less than one micron). The solar wind quickly eliminates the atoms and molecules, so that all the dissipation products are removed. And finally, I know of no strong streams that were not associated with known comets and near their orbits.

$H$. Alfvén: I don't think these points are decisive. A jet stream has an intrinsic tendency to contract; all this requires is that the collision time be smaller than the differential perturbation time. 
The important thing is, as Trulsen will describe, that the planetary perturbations produce a set of density waves in the stream. These density waves could then produce large concentrations in density at different points, especially if there is a series of consecutive perturbations, because the waves will be damped rather slowly.

B. Yu. Levin: Different parts of the stream move with different velocities, and if there is an increase in density, it is only temporary, when one part passes through another part, and the point of intersection is not suitable for the formation of a comet. I agree with Whipple that this process was possible in the early stages of the solar system but not at present.

$H$. Alfvén: The important thing is if the density goes above the limit where inelastic collisions become frequent. Condensation will then take place.

L. Kresák: I should like to point out that the spatial density of most well-known meteor streams is lower than that of the sporadic background through which they move. Even if planetary perturbations could build up a significant cloud-like concentration within a stream, it would certainly be quickly destroyed during the next revolution due to the dispersion of semimajor axes by the tangential velocity component. Moreover, we have ample evidence that the high-dispersion streams are old and the low-dispersion streams are new. This conclusion is based not only on the orbits, but also on such features as the lack of small particles within old streams, produced by interplanetary erosion and drag effects, or peculiar physical properties of the meteoroids of most recent origin (the Draconids).

H. Alfvén: We really don't know the density of meteor streams, because we can never know whether the Earth has passed through the centre of a stream. Moreover, in the case of a comet observed soon after its formation there should be a decrease in the density of the stream.

A. Z. Dolginov: How could comets, consisting mainly of ice, be formed of meteor particles? 